\title{
Large time off-equilibrium dynamics of a manifold in a random potential
}

\author{
Leticia F. Cugliandolo \\ Service de Physique de L'Etat Condensé, Saclay, CEA, Orme des Merisiers, 91191, Gif sur Yvette Cedex, France. \\ Jorge Kurchan and Pierre LgDoussal \\ CNRS-Laboratoire de Physique Theorique de l'Ecole Normale Superieurd, 24 rue Lhomond,75231 Cedex 05, Paris, France.
}

\begin{abstract}
We study the out of equilibrium dynamics of an elastic manifold in a random potential using mean-field theory. We find two asymptotic time regimes: (i) stationary dynamics, (ii) slow aging dynamics with violation of equilibrium theorems. We obtain an analytical solution valid for all large times with universal scalings of two-time quantities with space. A non-analytic scaling function crosses over to ultrametricity when the correlations become long-range. We propose procedures to test numerically or experimentally the extent to which this scenario holds for a given system.
\end{abstract}

The dynamics of an elastic manifold in a quenched random potential is relevant for a large number of experimental systems. Examples are flux lattices in highTc superconductors [3], interfaces in random fields [ [4, charge density waves [5], dislocation in disordered solids [6], surface growth on disordered substrates [7]. The competition between elasticity and disorder produces a 'glass' state that may exhibit pinning, slow dynamics and non linear macroscopic response (e.g, leading to zero linear resistivity in superconductors $[8]$ ). While there is a phenomenological picture based on scaling arguments (droplets) [3] no satisfactory analytical approach is available at present for the low-temperature dynamics.

The statics of a $d$-dimensional elastic manifold embedded in a $N$ dimensional space in the presence of a random potential was studied by Mézard and Parisi (MP) who applied a replica variational Gaussian approximation (Hartree) for $N$ finite which becomes exact at $N=\infty$ [9]. The replica symmetry breaking (RSB) solution captures some of the essential physics in finite $N$ dimension, such as sample-to-sample susceptibility fluctuations [9, 10], and predicts non trivial (Flory-like) roughness exponent $\zeta$. It allows for a theory [11] of the statics of the vortex glass state in superconductors relevant to experiments such as flux decorations. Other analytical approaches are based on RG methods [11 13] and it is as yet unclear whether they capture all the physics [14, 15]. Despite the obvious interest of the static approach, it applies by construction to equilibrium (Gibbs measure) properties, which may not hold for experimental times in a glassy system [16].

In this Letter we study, also within the Hartree approximation, the dynamics of this problem starting from a random configuration as in a temperature quench. We find that at low enough temperature there is an aging regime and that the system never reaches equilibrium. The correlation functions then depend not only on time differences but also on the waiting time after the quench. 
We obtain the two-time scaling with explicit space dependence, a new feature with respect to the mean-field analytical results for glassy systems obtained so far [17 20]. Details will be presented elsewhere [21].

This treatment is exact for $N=\infty$. At finite $N$ it holds a priori only within the Gaussian variational ansatz. Whether the properties hold qualitatively for true finite $N$ dimensional models cannot be answered analytically at present. The good qualitative agreement [17] of the MF dynamical analytical solution with experiments on spin glasses suggests that our results for $N=\infty$ may be relevant for some systems related to the present model.

The main purpose of this Letter is to suggest, on the basis of the exact solution for infinite $N$, definite predictions for the off-equilibrium dynamics which can be checked in numerical simulations and experiments. Our results provide a basis for a finite- $N$ analysis. Remarkably, despite the system being out of equilibrium, some of the results of the replica calculation by MP,e.g $\zeta$, are shown to carry through to the dynamics, albeit with a different interpretation in terms of directly observable timedependent physical quantities.

The model of a manifold of internal dimension $d$ embedded in a random medium of dimension $N$ is described, in terms of an $N$ component displacement field $\boldsymbol{\phi}, \boldsymbol{\phi}=\left(\phi_{1}, \phi_{2}, \ldots, \phi_{N}\right)$, by the Hamiltonian [9]

$$
H=\int d^{d} x\left[\frac{1}{2}(\nabla \phi(x))^{2}+V(\phi(x), \boldsymbol{x})+\frac{\mu}{2} \phi^{2}\right],
$$

$\mu$ is a mass, which effectively constraints the manifold to fluctuate in a restricted volume of the embedding space. $V$ is a Gaussian random potential with correlations

$$
\overline{V(\phi, \boldsymbol{x}) V\left(\boldsymbol{\phi}^{\prime}, \boldsymbol{x}^{\prime}\right)}=-N \delta^{d}\left(\boldsymbol{x}-\boldsymbol{x}^{\prime}\right) \mathcal{V}\left(\left(\boldsymbol{\phi}-\boldsymbol{\phi}^{\prime}\right)^{2} / N\right) .
$$

We consider the Langevin dynamics

$$
\frac{\partial \phi(\boldsymbol{x}, t)}{\partial t}=-\frac{\delta H}{\delta \phi(\boldsymbol{x}, t)}+\boldsymbol{\eta}(\boldsymbol{x}, t)
$$

with $\left\langle\eta_{\alpha}(\boldsymbol{x}, t) \eta_{\beta}\left(\boldsymbol{x}^{\prime}, t^{\prime}\right)\right\rangle=2 T \delta_{\alpha \beta} \delta^{d}\left(\boldsymbol{x}-\boldsymbol{x}^{\prime}\right) \delta\left(t-t^{\prime}\right)$. We let the system evolve from a spatially translationallyinvariant (STI) configuration at $t=0$. The system remains STI at subsequent times. We study the dynamics exactly for $N \rightarrow \infty$ and, for $N$ finite, within the dynamical Hartree approximation which amounts to substituting $\mathcal{V}$ by an effective $\hat{\mathcal{V}}[9,20]$. The 'equilibrium' dynamics (à la Sompolinsky [22]) was studied by Kinzelbach and Horner for general $d$ [23. The case $d=0$ has been studied in Ref. [18] and an analytical solution at large time was given in Ref. [20].

The quantities of interest in the large-time off - equilibrium dynamics are the two-time correlation $C_{x x^{\prime}}\left(t, t^{\prime}\right)$ $=1 / N \overline{\left\langle\phi(x, t) \cdot \phi\left(x^{\prime}, t^{\prime}\right)\right\rangle}$ and the response $R_{x x^{\prime}}\left(t, t^{\prime}\right)=$ $1 /\left.N \overline{\delta\langle\phi(x, t)\rangle / \delta \boldsymbol{f}\left(x^{\prime}, t^{\prime}\right)}\right|_{\boldsymbol{f}=0}$ where $\boldsymbol{f}\left(x^{\prime}, t^{\prime}\right)$ is a small perturbation applied at the space point $x^{\prime}$ at time $t^{\prime}$. We 
also define the mean squared displacement $D_{x x^{\prime}}\left(t, t^{\prime}\right)=$ $1 / N \overline{\left\langle\left(\phi(x, t)-\phi\left(x^{\prime}, t^{\prime}\right)\right)^{2}\right\rangle}$ and the correlation $B_{x x^{\prime}}\left(t, t^{\prime}\right)$ $=1 / N \overline{\left\langle\left(\phi(x, t)-\phi\left(x, t^{\prime}\right)\right)\left(\phi\left(x^{\prime}, t\right)-\phi\left(x^{\prime}, t^{\prime}\right)\right)\right\rangle}$. The brackets and overline represent the average over the thermal noise and the quenched disorder, respectively. The Fourier-transform $B_{k}$ and $R_{k}$ w.r.t. the space difference $x-x^{\prime}$ are used below, and we denote with tilde equal space $(x=0)$ two-time functions, $\tilde{B}=\int_{k} B_{k}$.

A common choice is $\mathcal{V}(z)=(\theta+z)^{1-\gamma} /(2(1-\gamma))$. 'Short-range' (SR) correlations correspond to $\gamma(1-$ $d / 2)>1$ and 'long-range' (LR) correlations to $\gamma(1-$ $d / 2)<1$. The static solution [9] is characterized by two exponents: in the LR case, an ultrametric ansatz gives $D_{x}^{s t}=\overline{(\phi(x)-\phi(0))^{2}} \sim x^{2 \zeta}$ with a roughness exponent $\zeta_{L R}=(2-d / 2) /(1+\gamma)$ and a free-energy fluctuation exponent $\theta$. In the SR case a one step RSB ansatz gives $\zeta_{S R}=(2-d) / 2$. Statistical rotational symmetry imposes in general $\theta=2 \zeta+d-2$. The $d=2$ Sine Gordon model is marginal and solved [11] by a one step RSB.

Aging. Let us describe the picture that emerges for large times and $\mu>0$. Equal-time quantities reach their asymptotic values which do not necessarily coincide with the equilibrium ones 17,21. For two-time quantities we consider two different regimes of the times.

(i) After a large waiting-time $t_{w}, B_{k}\left(\tau+t_{w}, t_{w}\right)$ first grows with $\tau$ in a manner independent of $t_{w}$, from 0 up to the Edwards-Anderson parameter for the mode $k$ defined as $b_{k}^{1} \equiv \lim _{\tau \rightarrow \infty} \lim _{t_{w} \rightarrow \infty} B_{k}\left(\tau+t_{w}, t_{w}\right)$. In this time regime the displacement is time-translation invariant (TTI); we denote it $b_{k}^{F}(\tau)=\lim _{t_{w} \rightarrow \infty} B_{k}\left(\tau+t_{w}, t_{w}\right)$. The response $r_{k}^{F}(\tau)=\lim _{t_{w} \rightarrow \infty} R_{k}\left(\tau+t_{w}, t_{w}\right)$ satisfies fluctuation dissipation (FDT) $r_{k}^{F}(\tau)=1 /(2 T) \partial_{\tau} b_{k}^{F}(\tau) \theta(\tau)$. The 'FDT-regime' is very much like an equilibration in a state, the manifold looks pinned with an effective mass $\bar{M}$, since $b_{k}^{1}=(2 T) /\left(k^{2}+\bar{M}\right)$ (for $\left.\mu \rightarrow 0\right)$.

(ii) However, for all $t_{w}$ and sufficiently large $\tau$, $B_{k}\left(\tau+t_{w}, t_{w}\right)$ continues to grow beyond $b_{k}^{1}$ up to $b_{k}^{o} \equiv$ $\lim _{\tau \rightarrow \infty} B_{k}\left(\tau+t_{w}, t_{w}\right)$. The growth of $B_{k}$ now depends on $t_{w}$ : the larger $t_{w}$ the slower the motion of the system, it ages. Thus in this model pinning is a gradual process, the older the system the more pinned it is but it is not pinned forever. The aging time-regime is thus defined (for $\left.t_{w} \rightarrow \infty\right)$ as the times $\tau$ such that $B_{k}\left(\tau+t_{w}, t_{w}\right)>b_{k}^{1}$. We denote by $b_{k}\left(\tau+t_{w}, t_{w}\right)$ and $r_{k}\left(\tau+t_{w}, t_{w}\right)$ the displacement and response in the aging regime, where both TTI and FDT are violated.

As regards the measurements of noise and susceptibility (i) corresponds to high frequencies while (ii) corresponds to low frequencies (scaling with the waiting time) such that noise and susceptibility depend on $t_{w}$. In a domain-growth process (i) corresponds to the fast thermal fluctuations of the spins around their mean magnetisation while (ii) corresponds to the actual growth of the domains. An important measurable quantity is the susceptibility $\chi_{k}\left(t, t^{\prime}\right)=\int_{t^{\prime}}^{t} d s R_{k}(t, s)$ of the mode $k$, i.e the total linear response to an external force of spatial modu- 
lation $k$ applied during the interval $\left[t^{\prime}, t\right]$. The results below imply $\chi_{k}\left(t_{w}+\tau, t_{w}\right)=k^{-2} F\left[k^{2} \chi_{0}\left(t_{w}+\tau, t_{w}\right)\right]$ where $\chi_{0}\left(t_{w}+\tau, t_{w}\right)^{-1}$ is a 'running' effective mass which exclusively depends on times through the local displacement, $\chi_{0}=\chi_{0}[\tilde{b}]$, and goes to zero at large time separation $\tau$. The typical internal distance $\sim \sqrt{\chi_{0}} \sim \tilde{b}^{1 / \zeta}$ grows slower with $\tau$ as the age $t_{w}$ increases.

We study $\mu \rightarrow 0$ after the large-times limit. If one takes $\mu=0$ from the start, one must take into account diffusion: $B_{x x}(t, 0) \rightarrow \infty$ at large $t$ since $\int d k b_{k}^{o}=\infty$. In addition to the aging regime defined as $t, t^{\prime} \rightarrow \infty$ with $B_{x x}\left(t, t^{\prime}\right)$ fixed, there is then a diffusion regime where $B_{x x}\left(t, t^{\prime}\right) / B_{x x}(t, 0)$ is fixed [21]. Our results for $B_{k}$ and $R_{k}$ are expected to hold also at $\mu=0$ in the aging regime.

Generalization of equilibrium theorems, two-time scalings. Let us describe in more detail the aging regime as derived below. For large times, the precise manner in which TTI is violated is described by 'triangle' relations [17] involving any three times:

$$
B_{k}\left(t_{\min }, t_{\max }\right)=f_{k}\left(B_{k}\left(t_{\min }, t_{\text {int }}\right), B_{k}\left(t_{\text {int }}, t_{\max }\right)\right)
$$

while the violation of FDT is given by:

$$
R_{k}\left(t, t^{\prime}\right)=X_{k}\left[B_{k}\left(t, t^{\prime}\right)\right] \partial_{t^{\prime}} B_{k}\left(t, t^{\prime}\right)
$$

where (2) means that $X_{k}$ depends on the times only through $B_{k}\left(t, t^{\prime}\right)$. In order to complete this ansatz, we have to specify how $f_{k}$ and $X_{k}$ for different $k$ are related to one another. This is done below in an algebraic manner, implying no new hypotheses with respect to the $d=0$ case $[17,20]$. We find that

$$
B_{k}\left(t, t^{\prime}\right)=B_{k}\left[k, B_{o}\left(t, t^{\prime}\right)\right]
$$

i.e. all $k$-modes depend on times only through the dependence of one of them. Thus one can use any two-time function, e.g $B_{o}\left(t, t^{\prime}\right)$ or $\tilde{B}\left(t, t^{\prime}\right)$ to parametrise the twotime dependence. We also show:

$$
X_{k}\left[B_{k}\right]=X_{k}\left[B_{k}\left[k, B_{o}\right]\right]=X_{o}\left[B_{o}\right]=\tilde{X}[\tilde{B}]
$$

i.e. the values of all $X_{k}$ are the same for times such that $B_{o}$ takes the same value. We have also defined $\tilde{X}\left[\tilde{b}\left(t, t^{\prime}\right)\right]=\tilde{r}\left(t, t^{\prime}\right) / \partial_{t^{\prime}} \tilde{b}\left(t, t^{\prime}\right)$. Eq. (何) implies that if $X$ is time independent in the aging regime, then it takes the same value for all $k$. Hence, for the $k$-mode susceptibility we have $\chi_{k}\left(t, t^{\prime}\right)=\chi_{k}\left[k, \chi_{0}\left(t, t^{\prime}\right)\right]$. These functional dependences are testable using two-time parametric plots. Their explicit forms for this model are determined below.

We find that: (i) in the FDT-regime $B_{k}<b_{k}^{1}$, $X_{k}\left[B_{k}\right]=-1 /(2 T)$ (ii) in the aging-regime $b_{k}^{0}>$ $b_{k}>b_{k}^{1}$ there are two distinct cases. For SR correlations $X_{k}\left(b_{k}\right)=X$ and $f_{k}$ has the form $f_{k}(u, v)=$ $\jmath_{k}^{-1}\left(\jmath_{k}(u) \jmath_{k}(v)\right)$. This implies $B_{k}\left(t, t^{\prime}\right)=\jmath_{k}^{-1}\left(h\left(t^{\prime}\right) / h(t)\right)$ where $h(t)$ is increasing and independent of $k$. For LR correlations $X_{k}\left(b_{k}\right)$ is a non-constant function of $b_{k}$. The function $f_{k}$ is $f_{k}(u, v)=\max (u, v)$. 
Formal solution for the spatial scaling. In order to extract the scaling properties of this model and to justify the above ansatz, we encode the correlation and response functions in the superspace order-parameter 25]. At the saddle point, using causality, $\boldsymbol{Q}_{x x^{\prime}}(1,2)$ reads

$$
\boldsymbol{Q}_{x x^{\prime}}(1,2)=C_{x x^{\prime}}\left(t_{1}, t_{2}\right)+\left(\bar{\theta}_{2}-\bar{\theta}_{1}\right)\left[\theta_{2} R_{x x^{\prime}}\left(t_{1}, t_{2}\right)+\theta_{1} R_{x^{\prime} x}\left(t_{2}, t_{1}\right)\right]
$$

The $\theta$ 's are Grassmann variables and we denote $1 \equiv$ $\left(t_{1}, \theta_{1}, \bar{\theta}_{1}\right), d 1 \equiv d t_{1} d \theta_{1} d \bar{\theta}_{1}, \boldsymbol{\delta}(1-2) \equiv\left(\theta_{2}-\theta_{1}\right)\left(\bar{\theta}_{2}-\right.$ $\left.\bar{\theta}_{1}\right) \delta\left(t_{2}-t_{1}\right)$ and $D^{(2)}(1) \equiv \partial_{\theta_{1}}\left(\partial_{\bar{\theta}_{1}}-\theta_{1} \partial_{t_{1}}\right)$. We use two types of functions of the super-order parameter: 'operator' as in $\boldsymbol{Q}_{x x^{\prime}}^{2}(1,2)=\int d 3 \boldsymbol{Q}_{x x^{\prime}}(1,3) \boldsymbol{Q}_{x x^{\prime}}(3,2)$ and 'pointwise' as in $\boldsymbol{Q}_{x x^{\prime}}^{\bullet 2}(1,2)=\left[\boldsymbol{Q}_{x x^{\prime}}(1,2)\right]^{2}$.

It is now easy to write the equation of motion for the order parameters, exact at the mean-field level:

$$
\left.\left(D^{(2)}-\nabla^{2}+\mu+\int d 3 \mathcal{V}^{\prime} \bullet\left(\boldsymbol{B}_{x x}(1,3)\right)\right) \boldsymbol{Q}_{x x^{\prime}}(1,2)-\delta^{d}\left(x-x^{\prime}\right) \boldsymbol{\delta}(1-2)-2\left[\mathcal{V}^{\prime} \bullet \boldsymbol{B}_{x x}\right) \boldsymbol{Q}_{x x^{\prime}}\right](1,2)=0
$$

where $\boldsymbol{B}_{x x^{\prime}}(1,2)=\boldsymbol{Q}_{x x^{\prime}}(1,1)+\boldsymbol{Q}_{x x^{\prime}}(2,2)-2 \boldsymbol{Q}_{x x^{\prime}}(1,2)$.

Using space translational-invariance one finds that all fourier modes $\boldsymbol{Q}_{k}$ can be expressed in terms of the zero mode $\boldsymbol{Q}_{k=0} \equiv \boldsymbol{Q}_{o}$ through the operator relation:

$$
\boldsymbol{Q}_{k}(1,2)=\left[k^{2} \boldsymbol{\delta}+\boldsymbol{Q}_{o}^{-1}\right]^{-1}(1,2) .
$$

This is (in an encoded notation) a scaling relation for the correlation and response functions involving the two times and spatial separation. Substituting Eq.(7) in Eq.(6) one can write a separate equation for each mode. Hence, we have mapped the problem of each $B_{k}\left(t, t^{\prime}\right)$, $R_{k}\left(t, t^{\prime}\right)$ into an effective $d=0$ problem with a complicated memory kernel [26]. Up to now we have made no approximations. A first important result is that one can now solve numerically the equation for one of the modes and then recover the space dependence algebraicly from Eq.(17). The above property implies that an ansatz as in problems without space-dependence [17,20] applies to each mode $B_{k}\left(t, t^{\prime}\right), R_{k}\left(t, t^{\prime}\right)$ and justifies Eqs. (12),(2).

Now, in the large time-limit any operator function $F\left[\boldsymbol{Q}_{A}\right]$ of an order parameter $\boldsymbol{Q}_{A}$ which can be parametrized by $f_{A}, X_{A}$, yields a new order parameter $\boldsymbol{Q}_{B}=F\left[\boldsymbol{Q}_{A}\right]$ which can also, at long times, be parametrized in the same form with $f_{B}, X_{B}$. The explicit computation of $f_{B}, X_{B}$ in terms of $f_{A}, X_{A}$ has been done in Ref. 19] and when applied to Eq. (7) yields explicit functional relations between $\boldsymbol{Q}_{k}, \boldsymbol{B}_{k}$ and $\boldsymbol{Q}_{0}$ and, in particular, Eqs. (3), (雨) for the components $B_{k}, R_{k}$.

One can extend this calculation to $O(N)$ spherically constrained models by letting $\mu$ be a function of time and by imposing $\boldsymbol{Q}_{x x}(1,1)=1$ for all $t_{1}$.

Explicit calculations. We can now establish the large time equations in both regimes. In the FDT-regime $r_{k}^{F}=$ $-x_{F} \partial_{\tau} b_{k}^{F}(\tau)$ with $x_{F}=-1 /(2 T)$ and one finds a single equation: 
$\frac{d b_{k}^{F}(\tau)}{d \tau}=2 T-\left(k^{2}+\bar{M}+4 x_{F} \mathcal{V}^{\prime}\left(\tilde{b}^{1}\right)\right) b_{k}^{F}(\tau)+4 x_{F} \frac{d}{d \tau} \int_{0}^{\tau} d \tau^{\prime} \mathcal{V}^{\prime}\left(\tilde{b}^{F}\left(\tau-\tau^{\prime}\right)\right) b_{k}^{F}\left(\tau^{\prime}\right)$

Neglecting the time derivatives in the l.h.s. of the full dynamical equations and integrating over the FDT regime (see 17,201) one finds the equations for the aging regime:

$$
\begin{aligned}
0= & r_{k}\left(t, t^{\prime}\right)\left(k^{2}+\bar{M}\right)+\frac{2 b_{k}^{1}}{T} \mathcal{V}^{\prime \prime}\left(\tilde{b}\left(t, t^{\prime}\right)\right) \tilde{r}\left(t, t^{\prime}\right)+4 \int_{t^{\prime}}^{t} d s \mathcal{V}^{\prime \prime}(\tilde{b}(t, s)) \tilde{r}(t, s) r_{k}\left(s, t^{\prime}\right) \\
0= & -b_{k}\left(t, t^{\prime}\right)\left(k^{2}+\bar{M}\right)+\frac{2 b_{k}^{1}}{T}\left(\mathcal{V}^{\prime}\left(\tilde{b}^{1}\right)-\mathcal{V}^{\prime}\left(\tilde{b}\left(t, t^{\prime}\right)\right)+4 \int_{0}^{t} d s\left(\mathcal{V}^{\prime}(\tilde{b}(t, s)) r_{k}(t, s)+\mathcal{V}^{\prime \prime}(\tilde{b}(t, s)) \tilde{r}(t, s) b_{k}(t, s)\right)\right. \\
& +2 T-4 \int_{0}^{t^{\prime}} d s\left(\mathcal{V}^{\prime}(\tilde{b}(t, s)) r_{k}\left(t^{\prime}, s\right)+\mathcal{V}^{\prime \prime}(\tilde{b}(t, s)) \tilde{r}(t, s) b_{k}\left(t^{\prime}, s\right)\right)-4 \int_{t^{\prime}}^{t} d s \mathcal{V}^{\prime \prime}(\tilde{b}(t, s)) \tilde{r}(t, s) b_{k}\left(s, t^{\prime}\right) .(10)
\end{aligned}
$$

$\bar{M} \equiv-4 \lim _{t \rightarrow \infty} \int_{0}^{t} d s \mathcal{V}^{\prime \prime}(\tilde{b}(t, s)) \tilde{r}(t, s)$ is the 'anomaly'. These equations have time-reparametrization invariance which prevent us from determining $h(t)$. The 'quasistatic' values $\tilde{b}^{1}, b_{k}^{1}$ follow from letting $t^{\prime} \rightarrow t_{-}$in Eq. 10):

$$
b_{k}^{1}=2 T /\left(k^{2}+\bar{M}\right)
$$

Similarly, Eq. (9) integrated over $k$, yields either the highT solution $\tilde{r}\left(t, t_{-}\right)=0$ or the low- $\mathrm{T}$ condition

$$
1=-4 \mathcal{V}^{\prime \prime}\left(\tilde{b}^{1}\right) \int_{k}\left(k^{2}+\bar{M}\right)^{-2}
$$

This implies $\tilde{b}^{1}=-4 T c_{d} /(d-2)\left(-4 c_{d} \mathcal{V}^{\prime \prime}\left(\tilde{b}^{1}\right)\right)^{(d-2) /(4-d)}$ and $\bar{M}=\left(-4 c_{d} \mathcal{V}^{\prime \prime}\left(\tilde{b}^{1}\right)\right)^{2 /(4-d)}$ for $d<4$, where $c_{d}=$ $\int_{k}\left(k^{2}+1\right)^{-2}$. Thus it is not necessary to know the details of the aging solution to determine $b_{k}^{1}, \tilde{b}^{1}$ and $\bar{M}$.

FDT regime. Defining $\phi(\tau)=4\left(\mathcal{V}^{\prime}\left(\tilde{b}^{F}(\tau)\right)-\mathcal{V}^{\prime}\left(\tilde{b}^{1}\right)\right)$, the Laplace transform w.r.t. $\tau$ of Eq. (\$) yields:

$$
b_{k}^{F}(s)=\left(x_{F} s\right)^{-1}\left(k^{2}+\bar{M}+s-x_{F} s \phi(s)\right)^{-1}
$$

At small $\tau, b_{k}^{F}(\tau) \sim\left(1-\exp \left(-A_{k} \tau\right)\right) /\left(x A_{k}\right)$ with $A_{k}=$ $k^{2}+\bar{M}-x_{F} \phi(0)$. When $b_{k}^{\text {int }} \sim 1 /\left(x A_{k}\right)$ there is a crossover to a slower regime where one can neglect the term $s(d / d \tau)$ and find a power law behaviour: $b_{k}^{F}(\tau)=$ $b_{k}^{1}-c\left(b_{k}^{1}\right)^{2} \tau^{-\beta}$ with $\beta$ determined by [23]:

$$
\Gamma[1-2 \beta] \Gamma[1-\beta]^{-2}=x_{F} Y\left(\tilde{b}^{1}\right)
$$

and $Y\left(\tilde{b}^{1}\right)=4 \mathcal{V}^{\prime \prime}\left(\tilde{b}^{1}\right)^{2} \mathcal{V}^{\prime \prime \prime}\left(\tilde{b}^{1}\right)^{-1} \partial_{\bar{M}} \ln \int_{k}\left(k^{2}+\bar{M}\right)^{-2}$. An explicit calculation gives $Y\left(\tilde{b}^{1}\right)=4 / \tilde{X}\left(\tilde{b}^{1}\right)$ in terms of the function $\tilde{X}(\tilde{b})$ defined by Eq. (19).

Aging regime in short range models. Power law models are short range for $\gamma>\gamma_{c}=2 /(2-d)$ and $d \leq 2$. With the ansatz $X_{k}\left[b_{k}\left(t, t^{\prime}\right)\right]=X$ Eqs.(9) and (10) reduce to a single equation for $b_{k}\left(t, t^{\prime}\right)$. One must have $X=-\bar{M} /\left(4 \mathcal{V}^{\prime}\left(\tilde{b}^{1}\right)\right)$. As discussed above $b_{k}\left(t, t^{\prime}\right)=$ $j_{k}^{-1}\left(h\left(t^{\prime}\right) / h(t)\right)$. Defining $u=\ln h(t)$ one has $b_{k}\left(t, t^{\prime}\right)=$ $\mathcal{B}_{k}\left(u-u^{\prime}\right)$ where $0<u<\infty$ and $\mathcal{B}_{k}(0)=b_{k}^{1}$ and $\mathcal{B}_{k}(\infty)=b_{k}^{0}$. We obtain:

$0=2 T-\left(k^{2}+\bar{M}+4 X \mathcal{V}^{\prime}\left(\tilde{b}^{1}\right)\right) \mathcal{B}_{k}(u)+4 X \frac{d}{d u} \int_{0}^{u} d u^{\prime} \mathcal{V}^{\prime}\left(\tilde{\mathcal{B}}\left(u-u^{\prime}\right)\right) \mathcal{B}_{k}\left(u^{\prime}\right)+4\left(X-x_{F}\right)\left(\mathcal{V}^{\prime}\left(\tilde{b}^{1}\right)-\mathcal{V}^{\prime}(\tilde{\mathcal{B}}(u))\right)$ 
Remarkably, this equation is formally similar to Eq. (8) though in a completely different variable. Laplace transforming w.r.t. $u$ one gets:

$\mathcal{B}_{k}(s)=b_{k}^{1}+(X s)^{-1}\left[\left(k^{2}+\bar{M}\right)^{-1}-\left(k^{2}+\bar{M}-X s \phi(s)\right)^{-1}\right]$

with $\phi(u)=4\left(\mathcal{V}^{\prime}(\tilde{\mathcal{B}}(u))-\mathcal{V}^{\prime}\left(\tilde{b}^{1}\right)\right)$.

At the beginning of the aging regime $u \ll 1$, we obtain $\mathcal{B}_{k}(u)=b_{k}^{1}-4 \mathcal{V}^{\prime \prime}\left(\tilde{b}^{1}\right)\left(k^{2}+\bar{M}\right)^{-2} u^{\alpha}$, and thus for $t^{\prime} \sim t$ :

$$
\tilde{b}\left(t, t^{\prime}\right)-\tilde{b}^{1} \sim \ln ^{\alpha}\left(h(t) / h\left(t^{\prime}\right)\right) \sim c\left(t_{w}\right)\left(\tau / t_{w}\right)^{\alpha}
$$

where $c\left(t_{w}\right)=\left(d \ln h\left(t_{w}\right) / d \ln t_{w}\right)^{\alpha}$. If $h(t)=t^{\delta}$ we recover the trap-model scaling where $c\left(t_{w}\right)$ is a constant [24]. The exponent $\alpha$ is determined by:

$$
\Gamma[1+2 \alpha] \Gamma[1+\alpha]^{-2}=X Y\left(\tilde{b}^{1}\right)
$$

For the power law model, $x Y\left(\tilde{b}^{1}\right)=(4-d) \gamma /(2(1+\gamma))$ which gives $\alpha \rightarrow 0$ when $\gamma \rightarrow \gamma_{c r}^{+}=2 /(2-d)$ and shows how ultrametricity in the case $\gamma<\gamma_{c r}$ is approached.

At widely separated times $u \rightarrow \infty$, the approach to $b_{k}^{o}$ is described by a scaling form of $k^{2} u$ :

$$
b_{k}\left(t, t^{\prime}\right)=b_{k}^{1}\left(1-\frac{x_{F}}{X}\right)-\frac{1}{X k^{2}}\left(1-\left(\frac{h\left(t^{\prime}\right)}{h(t)}\right)^{k^{2} /(X Z)}\right)
$$

where $Z=\int d u \phi(u)$ is a constant, finite for $\gamma>\gamma_{c r}$. Integrating over $k$ we also obtain the large time separation behaviour $\tilde{b}\left(t, t^{\prime}\right) \propto \ln ^{1-d / 2}\left(h(t) / h\left(t^{\prime}\right)\right)$.

Aging regime for long range models. These models are solved by the ultrametric ansatz $b_{k}\left(t, t^{\prime}\right)=$ $\max \left(b_{k}(t, s), b_{k}\left(s, t^{\prime}\right)\right)$, which inserted in (9), (10) leads [21] to a single equation parametrized by $b$

$$
\chi_{k}\left(t, t^{\prime}\right)=\chi_{k}[\tilde{b}]=\left(k^{2}+\bar{M}-\bar{M}(\tilde{b})\right)^{-1}
$$

after simple manipulations as in 20]. We have defined $\bar{M}[\tilde{b}]=-4 \int_{\tilde{b}}^{\tilde{b}_{k}^{1}} \mathcal{V}^{\prime \prime}\left(\tilde{b}^{\prime}\right) \tilde{X}\left[\tilde{b}^{\prime}\right] d \tilde{b}^{\prime}$ and used (2)-(四). Taking a derivative w.r.t. $\tilde{b}$, using $\partial_{\tilde{b}} \chi_{k}\left[b_{k}\right]=\tilde{X}[\tilde{b}] \partial_{\tilde{b}} b_{k}$, dividing by $\tilde{X}[\tilde{b}]$ and integrating over $\tilde{b}$, one gets:

$$
b_{k}=b_{k}^{1}+\int_{\tilde{b}}^{\tilde{b}^{1}} d \tilde{b}^{\prime} \frac{4 \mathcal{V}^{\prime \prime}\left(\tilde{b}^{\prime}\right)}{\left(k^{2}+\bar{M}-\bar{M}\left(\tilde{b}^{\prime}\right)\right)^{2}}
$$

It also implies the self-consistency condition $1=$ $4 \mathcal{V}^{\prime \prime}(\tilde{b}) \int_{k}\left(k^{2}+\bar{M}-\bar{M}(\tilde{b})\right)^{-2}$ which coincides with the marginality condition for the replicon. One also obtains

$$
\tilde{X}(\tilde{b})=-a_{d} \mathcal{V}^{\prime \prime \prime}(\tilde{b})\left(-\mathcal{V}^{\prime \prime}(\tilde{b})\right)^{2(d-3) /(4-d)}
$$

with $a_{d}=(4-d)\left(4 c_{d}\right)^{2 /(4-d)} / 2$. This result, derived in a rather simple way, is formally identical to the result of the statics [9] and is here shown to apply directly to the off-equilibrium dynamics. The self-energy of the statics $[\sigma](u)$ is thus formally identified with $\bar{M}-\bar{M}(\tilde{b})$. 
One general prediction for the aging regime of manifolds is the existence of a scaling form for $b_{r}\left(t, t^{\prime}\right)$ as a function of $r$ and the two-times quantity $\tilde{b}\left(t, t^{\prime}\right)$ :

$$
b\left(r, t, t^{\prime}\right)=b_{r=0}\left(t, t^{\prime}\right) H\left[r / b_{r=0}\left(t, t^{\prime}\right)^{\frac{1}{2 \zeta}}\right]
$$

which holds at large scales, i.e large $r$ and large, widely separated times. One has $H[0]=1$. The Hartree method yields a remarkably simple analytical form. Using 18 one finds [21] $\partial_{\tilde{b}} b_{k}=-4 \mathcal{V}^{\prime \prime}(\tilde{b}) /\left(k^{2}+\left(-4 \mathcal{V}^{\prime \prime}(\tilde{b}) c_{d}\right)^{\frac{2}{4-d}}\right)^{2}$, e.g in real space in $d=3$ one has $\partial_{\tilde{b}} b_{r}=\exp \left(\mathcal{V}^{\prime \prime}(\tilde{b}) r /(2 \pi)\right)$.

In conclusion, we described, using the Hartree approximation, the features of aging of a manifold in a random medium. This provides a frame of reference for the analysis of data from experiments and simulations on realistic systems and will allow to determine in each case whether such a regime exists and up to what times.

[1] Electronic address: leticia@amoco.saclay.cea.fr

[2] LPTENS is a Laboratoire Propre du CNRS, associe a l'Ecole Normale and Universite de ParisSud Electronic address: kurchan@physique.ens.fr, ledou@physique.ens.fr.

[3] for a review see G. Blatter et al., Rev. Mod. Phys. 66 1125 (1994).

[4] T. Nattermann and P. Rujan; Int. J. Mod. Phys. B3 1597 (1989).

[5] H. Fukuyama and P.A. Lee, Phys. Rev. B 17535 (1978)

[6] V.M. Vinokur, J. Phys. France 471425 (1986).

[7] D. Cule and Y. Shapir; Phys. Rev. Lett. 74, 114 (1995).

[8] M. Feigelman, V.B. Geshkenbein, A.I. Larkin and V. M. Vinokur, Phys. Rev. Lett. 632303 (1989). D. S. Fisher and M. P. A. Fisher and D. A Huse, Phys. Rev. B 43130 (1990).

[9] M. Mézard and G. Parisi; J. Phys. I (France) 1, 809 (1991).

[10] T. Hwa and D.S. Fisher; Phys. Rev B49 3136 (1994).

[11] P. Le Doussal and T. Giamarchi; Phys. Rev. B52 1242 (1995).

[12] J. Cardy and S. Ostlund; Phys. Rev. B25 6899 (1982). Y. Y. Goldschmidt and B. Schaub; Nucl. Phys. B251, 77 (1985). Y-C Tsai and Y. Shapir; Phys. Rev. Lett. 69, 1773 (1992).

[13] L. Balents and D. S. Fisher; Phys. Rev. B48, 5949 (1993).

[14] P. Le Doussal and T. Giamarchi; Phys. Rev. Lett. 74, 606 (1995). J. Kierfeld (to be published).

[15] it is puzzling that FDT is usually assumed in dynamical RG.

[16] L. C. E. Struik; 'Physical aging in amorphous polymers and other materials', Elsevier, Houston (1978). L. Lundgren, P. Svedlindh, P. Nordblad and O. Beckman; Phys. Rev. Lett. 51, 911 (1983). E. Vincent, J. Hammann and M. Ocio; in 'Recent progress in Random Magnets', ed. D. H. Ryan, World Scientific, Singapore (1992). 
[17] L. F. Cugliandolo and J. Kurchan; Phys. Rev. Lett. 71, 173 (1993); J. Phys. A27, 5749 (1994); Phil. Mag. B71, 501 (1995).

[18] S. Franz and M. Mézard; Europhys. Lett. 26, 209 (1994); Physica A209, 1 (1994).

[19] A. Baldassarri, L. F. Cugliandolo, J. Kurchan and G. Parisi; J. Phys. A28, 1831 (1995).

[20] L. F. Cugliandolo and P. Le Doussal; Phys. Rev. E (to be published).

[21] L. F. Cugliandolo, J. Kurchan and P. Le Doussal (to be published).

[22] H. Sompolinsky and A. Zippelius; Phys. Rev. Lett. 45, 359 (1981); Phys. Rev. B25, 274 (1982). H. Sompolinsky; Phys. Rev. Lett. 47, 935 (1981).

[23] H. Kinzelbach and H. Horner; J. Phys. I (France) 3, 1329 (1993), ibid 3, 1901 (1993).

[24] J.-P. Bouchaud; J. Phys. I (France) 2, 1705 (1992). J.P. Bouchaud and D.S. Dean; J. Phys. I (France) 5, 265 (1995).

[25] J. Kurchan; J. Phys. I (France) 2, 1333 (1992).

[26] a qualitative reduction of the problem of a driven manifold onto a correlated $d=0$ problem is used in P. Le Doussal, V. M. Vinokur, to be published. 